\title{
RADIO OCCULTATION ON THE VENERA-D MISSION: A CONCEPT OF RADIO FREQUENCY SUBSYSTEM AND RADIO SCIENCE TECHNIQUE
}

Anatoly L. Gavrik, Sergey F. Kolomiets, Mikhail I. Bondarenko, Lyudmila A. Lukanina, Tatiana F. Kopnina

Kotelnikov Institute of Radioengineering and Electronics of RAS, Fryazino branch, http://fire.relarn.ru Fryazino 141190, Moscow region, Russian Federation

Yaroslav A. Ilyushin

Lomonosov Moscow State University, Physical Faculty, Atmospheric Physics Department, http://atm563.phys.msu.ru Moscow 119991, Russian Federation

Kotelnikov Institute of Radioengineering and Electronics of RAS, Fryazino branch, http://fire.relarn.ru Fryazino 141190, Moscow region, Russian Federation

alg248@hotmail.com, bottom@mail.ru,ilyushin@physics.msu.ru,linuxoid2013@mail.ru, lukaninal@hotmail. com, tkopnina@hotmail.com

Abstract. Main goals of radio-occultation experiments in VENERA-D mission are formulated. It is shown, based on the results of radio-occultation experiments obtained earlier with high potential radio-links in the Venusian gaseous envelop, that using variations of power and frequency at two coherent radio waves, one can study a fine structure of refracting medium which may bring fundamentally new information about the atmosphere-ionosphere system of Venus. Given the accuracy of data interpretation depends on different high-frequency subsystem performance characteristics, it is shown that the prime direction of the development, must be the balanced increase of the energy potential of radio links and improving the stability of the oscillators used. A novel technique of data interpretation applicable to high potential measurements in the ionosphere and atmosphere of the planet is discussed. Among all the other things the suggested technique makes it possible to see and study propagation of disturbances from the atmosphere into the ionosphere. Moreover, it is shown that the correlation between amplitude and frequency of signals which is underpinning the technique mentioned can be seen as an effective criterion of the validity of geometrical optics spherically symmetrical approximations for the inverse problem solution.

Keywords: wave propagation, occultation, ionosphere and atmosphere of Venus

UDC 535.37:523.42

Bibliography - 15 references

Received 17.04.2019, accepted 23.04.2019

RENSIT, 2019, 11(1):5-12

DOI: $10.17725 /$ rensit.2019.11.005

\section{Contents}

1. INTRODUCTION (5)

2. TASKS OF RADIO OCCULTATION EXPERIMENTS IN THE VENUS-D MISSION (6)

3. Methods of Radio occultation experiment (7)

4. Data Processing Technique (8)

5. Diagnostics of LaYered structures in the IONOSPHERE (9)

6. Conclusion (11)

REFERENCES (11)

\section{INTRODUCTION}

For several decades, radio occultation sounding has been developed as a diagnostic technology for the gas envelopes of space objects: the Earth, planets, comets and theSun [1]. Radio-obscuration studies on Venus began in 1967 [2]. Thanks to the successful missions of the spacecraft (SC) MARINER-5,-10, VENUS-9,-10, PIONEERVENUS, VENUS-15,-16, MAGELLAN, VENUS-EXPRESS, AKATSUKI, more than 1500 radio transmissions of the Venosphere and Venus atmosphere were conducted [3-9]. The amount of radio sounding data accumulated to date is relatively small ( $\sim 1500$ sessions), while the amount of radio sounding of the Martian 
gas envelope exceeds 6000, the number of radio sounding of the near-earth shell is several thousand per day and several million sessions have been conducted.

Using the available experimental data, the structural characteristics of the ionosphere and the upper atmosphere of Venus were investigated and described in the literature [1-9]. These results cover a wide range of altitudes above the surface of the planet and solar activity. It is important to say that, although much is known about the Venus ionosphere, our understanding remains rather superficial in most aspects. Venus, like the Earth, is a complex world, and in order to take another step towards its adequate understanding, the planned mission of VENER-D [10] should focus on the issue of obtaining new data that are more likely to lead to new knowledge, and not only to the accumulation of measurement statistics using known tools and data analysis methods.

In the VENERA-D mission [10], our interest is focused on small-scale (from $0.5 \mathrm{~km}$ to $10 \mathrm{~km}$ ) features of the ionosphere and atmosphere, as well as on processes that affect their structure. The most attractive are the least studied questions: the interaction between the lower ionosphere and the upper atmosphere of Venus, as well as wave processes both in the atmosphere and in the ionosphere. The experience of previous missions shows that, having sufficient energy potential of radio lines, it is possible to reveal the properties of all layers of the ionosphere [11], including by comparing radio occultation data with direct measurements of sensors installed on the orbital apparatus of the VENER-D project.

The analysis of radio-sounding data is usually performed in the approximation of geomeric optics, and the dispersion interferometer method is used to separate radio effects from the ionosphere and atmosphere. The known integral relations describing the corresponding formulation of the inverse problem allow us to calculate the radial profiles of the electron concentration in the ionosphere, density and temperature in a neutral atmosphere from changes in the frequency or power of the probing signals [1]. However, questions about the accuracy of the radio sounding method in determining the parameters of the atmosphere and the ionosphere are still relevant [12, 13], and the issues of extracting information about the gas envelope from experimental data require improving measurement techniques and analyzing experimental results [14].

The experience of all previous missions to Venus allows us to choose the optimal conditions for radio occultation experiments. The improved data processing model discussed in this article, applicable to the results of highpotential measurements, being the basis of the experiment, will provide new reliable data on the night ionosphere, on the lower part of the daytime ionosphere and on the propagation of disturbances from the atmosphere into the ionosphere. As a result, radio sounding using equipment installed on an orbiter in the VENER-D mission can be considered as one of the most inexpensive and reliable ways to obtain unknown and important information about the gas envelope of Venus.

\section{TASKS OF RADIO REPLACEMENT EXPERIMENTS IN THE VENER-D MISSION}

Radiophysical studies in the VENERA-D mission can be carried out with coherent twofrequency radiation in the $X(3.6 \mathrm{~cm})$ and $S(13.7 \mathrm{~cm})$ bands with a high potential of radio lines. Three categories of experiment and observation are possible. First, the study of the planetary atmosphere and ionosphere. These measurements can be performed both with direct (implying that the transmitters are on board), and with reverse (transmitters on Earth) translucency. Secondly, studies of the so-called "bistatic scattering" properties, when the receiving station on Earth registers not only the direct signal from the spacecraft, but also 
the signal reflected from the surface of Venus. Thirdly, the study of the properties of the solar wind and the solar corona using direct radio sound transmission with small periods of signal sampling, including its synchronous reception by several ground stations (interferometric reception). Thus, the following studies will be possible:

Obtain electron concentration data in the altitude range from $\sim 70 \mathrm{~km}$ to $\sim 1000 \mathrm{~km}$ and obtain data on temperature, density, and pressure in the atmosphere in the altitude range from $\sim 40$ $\mathrm{km}$ to $\sim 100 \mathrm{~km}$ as a function of altitude, latitude, local time, and season.

The study of the dynamics of multilayer structures in the atmosphere and ionosphere of Venus, their connection with wave processes, solar activity and the flow of the solar wind.

Identification of links between processes in the ionosphere, the thermosphere and the lower atmosphere on the day and night side of Venus.

The study of the scattering properties of the surface of Venus, the dielectric constant and density of the soil, identifying areas with abnormal scattering coefficients.

Study of the dynamics of small-scale and large-scale inhomogeneities of interplanetary and near-solar plasma.

The study of the propagation of radio waves in space environments.

\section{TECHNIQUE OF RADIO- CHANGING EXPERIMENT}

The radio sounding scheme of the gas envelope of the planet is shown in Fig. 1. At

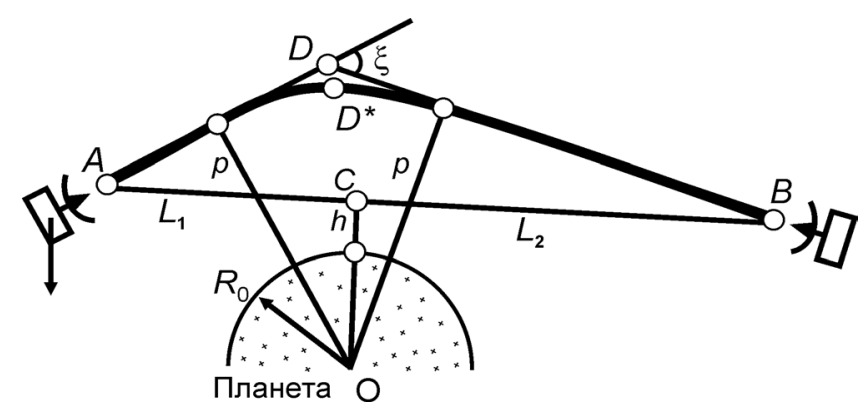

Fig. 1. Scheme of radio occultation experiment. The transmitter is atpoint $A$, the receiver is at point $B$. The curvature of the radio path $A D^{*} B$ is due to the refraction of the radio wave in the gas envelope. the beginning of the experiment, the radio path between the source and receiver of the signals passes above the ionopause. The orbiting object $A$ emits signals received by the object $B$ (the radial approximation relations used below are independent of the direction of radiation). It is assumed that the speed of the line of sight $A B$ in the shell of the planet $V=d h / d t$ ( $b$ is the height of the line $A B$ above the surface of the planet), as well as the distances $C A$ and $C B$ from the pericenter of the line $\mathrm{AB}$ to the radiator $L_{1}$ and receiver $L_{2}$ during the sensing process change slightly so the change of these parameters in the experiment can be neglected. In a spherically symmetric medium, the asymptotes $A D$ and $D B$ of the curved radio beam $A D^{*} B$ have an impact parameter $p(t)$, the value of which, due to the small angle of refraction $\xi(t)$, is equal to the distance $O D^{*}$ of the beam turning point. Such a model is permissible when probing the near-planetary environment of Venus above the cloud layer.

The transmitter KA emits two continuous coherent radio waves in the process of moving, the measuring system uses only limited portions of the radio field that fall into the aperture of the receiving antenna on the measurement time intervals $\tau$. The receiver determines the average for the time interval $\tau$ the power of each signal, which is then normalized to the signal power level in the control area in the absence of the medium being probed. The recorded power variations of the signal $X a p(t)=X a(t)+X p(t)$ are the sum of the changes in plasma power $X p(t)$ and in the neutral medium $X a(t)$, which depend only on the deformation of the ray tube in the absence of absorption and scattering of radio waves. Near the lower boundary of the ionosphere it is unambiguous to separate $X p(t)$ and $X a(t)$ from the $X a p(t)$ function [14]. The receiver also determines the increments for the time $\tau$ of the phase $\varphi(t)$ of each signal and calculates the instantaneous frequency $f(t)$ $=(2 \pi)^{-1} d \varphi(t) / d t$. The change in the frequency of the signal $f a p(t)=f a(t)+f p(t)$ is due to the 
change in the angle of refraction $\xi(t)$ when the radio beam is immersed in the shell of the planet containing neutral and ionized components (terms $f a(t), f p(t))$. To isolate the plasma effect, the difference frequency $\delta f(t)$ is calculated from data on the frequency variations of the received coherent signals $f_{D}(t)$ and $f_{C}(t)$ :

$\delta f(t)=q^{2} \cdot\left(q^{2}-1\right)^{-1} \cdot\left[f_{D}(t)-f_{C}(t) \cdot q^{-1}\right]$.

In the case of rectilinear propagation of radio waves, $\delta f(t)$ coincides with a change in the frequency of the low-frequency signal in the plasma $\not p(t)$ ( $q$ is the ratio of the high radiated frequency to the low frequency). In the Venus probed ionosphere, the trajectories of signals of different ranges pass at different heights, therefore $\not p(t)$ differs from $\delta f(t)$. A technique has been developed to restore $\not p(t)$ from variations $\delta f(t)$, the error in determining $f p(t)$ does not exceed the amount of instrumental noise $\left(10^{-2} \ldots\right.$ $3 \cdot 10^{-3} \mathrm{~Hz}$ at $\left.\tau=1 s\right)[6]$.

The experience of previous missions shows that the dynamic range of the radio links used must be at least $45 \mathrm{~dB}$ and be balanced. The latter is no less important for reliable data interpretation and requires ensuring the potential of both lines is high enough to prevent the noise of one of them from dominating the final results. Radio measurements in the VENERA-D mission imply the use of ground antennas with diameters of 70 and $64 \mathrm{~m}$, and hydrogen maser receivers assembled according to a scheme that involves parallel implementation of recording signals in the $X(3.6 \mathrm{~cm})$ and $S(13.7 \mathrm{~cm})$ bands, as in a narrow band (with phase locked loop), and in a wide band. The latter allows one to expect reliable and interpretable signal registration, when its power is rather low for normal operation of the phase tracking subsystem.

To perform all the studies listed above, there must be a transmitter in the $X$-range of more than $30 \mathrm{~W}$ and no less than $40 \mathrm{~W}$ of the transmitter in the $S$-range. Both of them are expected to be well stabilized and effectively shielded to provide the required stability over time intervals from $0.05 s$ to $600 s$, which correspond to the typical duration of signal sampling periods in various radio-dark experiments. Considering that short-term frequency deviations can be directly projected onto the fine structure of the planetary environment, it is advisable to use on-board local oscillators with a frequency stability value no worse than $10^{-12}$ for a time interval of up to $10 \mathrm{~s}$. Thus, the main direction of development of a radio-technical complex that performs radio sounding experiments is to increase the energy potential of a radio link and to improve the stability of the equipment used, since the accuracy of the information in the interpretation of these radio insights depends on the relationship between the radiophysical effects caused by the medium under study and the interfering factors.

\section{DATA PROCESSING TECHNIQUE}

The accuracy of determining the parameters of the atmosphere and ionosphere in solving the inverse problem of radio sounding is unsatisfactory in the region of the lower boundary of the ionosphere at altitudes from 75 $\mathrm{km}$ to $120 \mathrm{~km}$ [11]. To study the atmosphereionosphere system, a method using the main merit of radio sounding is necessary: continuous (within $2 \ldots 5 \mathrm{~min}$ ) recording variations of the refraction angle $\xi(t)$ from which the refractive index profile $n(b)$ is calculated along the vertical from the upper ionosphere to the cloud layer the atmosphere. The ways to solve the inverse problem of radio sounding of the atmosphere and the ionosphere are different, but the principle of determining $n(b)$ is based on the integral connections of the functions $f a(t), X a(t)$, $f p(t), X p(t)$ with the parameters of the medium being probed [15]. In [11], it was shown that the information obtained on the solution of the inverse problem of small variations $n(b)$ is not always reliable. In particular, near the lower boundary of Venus's ionosphere in a region of $\sim 40 \mathrm{~km}$ (in the altitude range $75 \ldots 120 \mathrm{~km}$ ), the insufficient accuracy of determining $n(b)$ in the atmosphere and ionosphere limits the possibility of studying stratified layers. 
The developed new method [11] uses the relation (1) to separate the effects of the atmosphere and the ionosphere, which distinguishes the plasma effect $\delta f(t)$. Using $\delta f(t)$, we calculate the frequency change due to the influence of the ionosphere $f p(t)$, then from the function $f_{D}(t)$ we determine the influence of the atmosphere $f a(t)$. The functions $f a(t), X a(t), f p(t)$, $X p(t)$ are related to the refractive index $n(b)$ of the medium being examined and the angle of refraction $\xi(t)$ by well-known relations [15]. For a stratified non-absorbing radio wave medium having the property of spherical symmetry in a solid angle less than $10^{\circ}$, it is possible to obtain approximate formulas by eliminating from the multiparameter relations given in [15] all the terms whose contribution does not exceed random fluctuations. In this case, with $L_{2}>>L_{1}$ and a small parameter $\xi(t)$, the following relations are valid:

$X a p(t)=\left\{1-L_{1} \cdot d \xi(t) / d p\right\}^{-1}$,

$p(t)=R_{0}+h(t)+L_{1} \cdot \xi(t)$,

$f a p(t)=\xi(t) V \cdot L_{i} \cdot \lambda^{-1}$,

where $R_{0}$ is the radius of the planet, $\lambda$ is the length of the radio wave. The error of formulas (2) - (4) with $\xi(t)<10^{-3} \mathrm{rad},|n(b)-1|<10^{-4}$ does not exceed the distortion caused by noise. Differentiation of formulas (3), (4) and their use to transform formula (2) allows to obtain the following relations:

$d p(t) / d t=V \cdot X a p(t)$.

$X a p(t)=1+\left[\lambda \cdot L_{1} \cdot V^{-2}\right] \cdot d f a p(t) / d t$.

Formula (5) establishes a connection between the vertical component $V$ of the speed of spacecraft and the speed of immersion of a curved radiobeam into the shell of the planet $d p(t) / d t$, this connection was previously detected experimentally, but the limits of applicability of the empirical formula in real experiments in studying thin layers remained unclear. The formula (5) obtained by the transformations of the known relations (2) - (4) proves the validity of its use for arbitrary variations of $n(b)$ that do not violate the limitations of the ray approximation.
The relation (6) opens up prospects for solving new problems of diagnosing the atmosphere-ionosphere system. Using the nonenergy signal parameters $f a(t), f p(t)$, one can calculate the predicted refractive changes by formula (6) and compare them with the energy parameters of the signals $X a(t), X p(t)$. The presence of correlation between measured and calculated parameters will allow to distinguish small variations of $n(b)$ in the stratified layers of the atmosphere-ionosphere system against the background of similar-shaped interference, since random fluctuations of energy and nonenergy parameters are statistically independent. The lack of correlation between variations of the measured and calculated parameters will indicate the dominant influence of noise and/or the inapplicability of the approximations used.

\section{DIAGNOSTICS OF LAYERED STRUCTURES IN THE IONOSPHERE}

The proposed method for analyzing radiosounding data will provide important information on the propagation of disturbances in the gas envelope in the new mission to Venus. A reliable criterion for detecting stratified layers in the atmosphere is the coincidence of $X a(t)$ variations with measured functions $X_{D}(t)$ or $X_{C}(t)$, and in the ionosphere the coincidence of $X p(t)$ variations with $X_{D}(t)$ or $X_{C}(t)$. In addition, such coincidences will confirm the validity of applying the ray approximation and spherical symmetry in solving the inverse problem, since relation (6) is satisfied only within the framework of the adopted model. In experiments, one can observe a correlation of pulsations $X a(t)$, $X p(t), X_{D}(t), X_{C}(t)$, which do not coincide in amplitude. In this case, the studied effects are masked by interfering factors, which include: an insufficiently short data sampling interval $\tau$, signal fluctuations due to noise or turbulence of the medium, absorption or scattering of radio waves, violation of the applicability of radiation approximation or spherical symmetry. In such cases, the interpretation of the data will require 
careful analysis of possible sources of error and justification of the accuracy of the information obtained.

Using the data of the VENER-15,-16 missions and the original data processing methods, we finally managed to form a solid experimental basis for the above theoretical relationships and show that in the field of application of geometric optics, the energy flux density of signals in a medium with spherical symmetry changes in direct proportion wave vector [11]. This means that fluctuations of the signal power in experiments on radio sounding of the atmosphere and the ionosphere are directly correlated with the rate of change of frequency, since the medium can be considered as spherically symmetric due to the influence of the gravitational field. At the same time, true stochastic fluctuations (noise) of power and frequency are not correlated. Considering that this is a new way of presenting signals, it opens up prospects for the application of new data processing technology, which we hope will allow deeper penetration into the ionosphere and more reliably explore the processes of interaction between the atmosphere and the ionosphere, including the propagation of wave processes from the atmosphere into the ionosphere.

The effect underlying this study can be found in a spherically layered medium if the layers are sufficiently dense and the level of hardware noise is low, and diffraction and absorption of electromagnetic waves are absent. That is why the data from the VENUS-15,-16 missions, which operated at higher power and at a lower frequency than most other missions, were critically needed to test the concept of a new data processing technology. The most intriguing result obtained using data from the VENUS-15,-16 spacecraft is that the layered structures of the Venusian ionosphere are found and studied at extremely low altitudes $80 . . .120$ $\mathrm{km}$ above the surface of the planet, where the electron concentration is extremely small compared to the error of its determination in
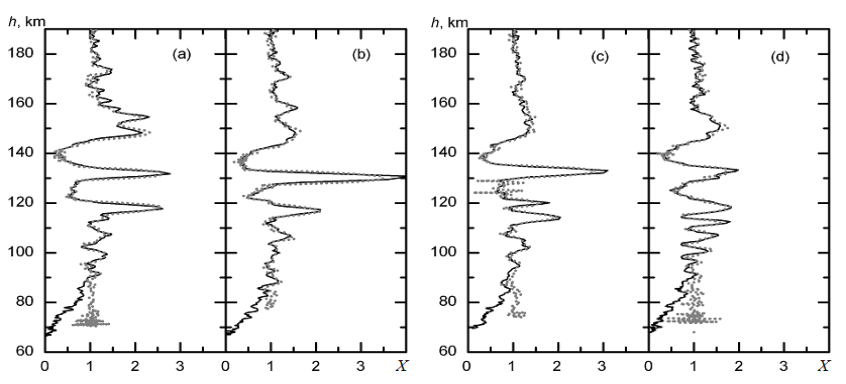

Fig. 2. Comparison of the refractive power change $X_{0}(b)$ of a signal with a wavelength of $32 \mathrm{~cm}$. Venus-15 spacecraft, -16 (solid curve) with the refractive change $X_{1}(b)$ (gray dots) calculated from frequency data in four radio-echo sounding sessions. The vertical axis is the height of the radio beam above the surface of Venus.

the case of applying the traditional method of solving the inverse problem of radio sounding.

Typical stratified periodic variations of power $X_{0}$ and $X_{1}$ are presented in Fig. 2 for four sessions of radio sounding. The idea of detecting ionospheric disturbances is to compare two diagrams with signal power values ( $X$ axis) along altitudes ( $Y$ axis), while one of them $\left(X_{0}\right)$ represents experimentally observed variations and fluctuations in signal power due to the influence of the ionosphere and atmosphere and the other $\left(X_{1}\right)$ means "prediction" of the same parameter calculated using relation (6) based on variations in the signal frequency in the ionosphere. It can be seen in Fig. 2 is a good match between the $X_{0}$ and $X_{1}$ diagrams in the altitude range of 90 to $180 \mathrm{~km}$. Fluctuations above $180 \mathrm{~km}$ have a pure stochastic character, which indicates that this altitude interval does not contain detectable ionized layers with stable deviations of the average refractive index. As follows from Fig. 2, below $85 \mathrm{~km}$, the plasma layers are also absent, and the attenuation of power during refraction in the atmosphere rapidly increases.

The variations of $X_{1}(b)$ in Fig. 2 due to the rarefaction or concentration of the beam beams with a change in the refractive index in the ionosphere. At altitudes from 90 to $160 \mathrm{~km}$, there is a correlation of $X_{0}(b)$ with $X_{1}(b)$, reaching values of 0.9 , which indicates the influence of the plasma layers and the absence of the influence 
of the atmosphere. The most important is the detection of alternation of maxima and minima of $X_{0}(b)$ below $120 \mathrm{~km}$, coinciding with $X_{1}(b)$, which indicates the presence of numerous stratified ionized structures in the Venusian lower daytime ionosphere. In foreign experiments on the radio sounding of the Venus ionosphere $[2,3,5,7,8,9]$, this effect was almost imperceptible due to the limited accuracy of the technique used. The detected periodic oscillations $X_{1}(b)$, which coincide with the oscillations $X_{0}(b)$, indicate the regular existence of stratified periodic perturbations $n(b)$ due to fluctuations in the electron concentration in the lower boundary of the Venus ionosphere. This circumstance indicates the possibility of studying the interaction between the lower ionosphere and the upper atmosphere of Venus, and also opens up the prospect of studying wave processes both in the atmosphere and in the ionosphere in radio sound experiments of the planned VENER-D mission.

\section{CONCLUSION}

To solve the fundamental problems of comparative planetology in the VENERA-D project, it is proposed to perform multiple radioecho sounding of the Venosphere ionosphere and atmosphere, sounding of the surface of Venus using the method of bistatic radar, radio-sounding of the interplanetary medium and the near-solar plasma when Venus sets behind the Sun. The most important are the least studied issues: the interaction between the lower ionosphere and the upper atmosphere of Venus, as well as wave processes both in the atmosphere and in the ionosphere. The experience of previous missions showed that, having sufficient energy potential of radio lines, it is possible to reveal the properties of all layers of the ionosphere, including by comparing radio occultation measurements and data collected from instruments installed on the VENERA-E orbiter. It is shown that an improved data processing model applicable to high-potential measurements, being the basis for the experiment, will provide new reliable information about the night ionosphere, the lower part of the daytime ionosphere and the distribution of disturbances from the atmosphere into the ionosphere. The presented analysis of radio-radiation data in the mission Venus-15,-16 indicates that the ionized layers constantly exist not only in the lower ionosphere of the Earth (in region $D$ ), but also in the daytime ionosphere of Venus. A deeper investigation of the nature of such disturbances will be possible in the mission of VENERA-D. The proposed approach will allow us to study the fine structure of the interaction of the atmosphere and the ionosphere using variations in the power and frequency of two coherent signals. Optimization of the radio frequency subsystems on the basis of summarizing the experience of previous missions will effectively apply modern approaches to obtain fundamentally new information about the atmosphere and ionosphere of the planet, as well as about the state and dynamics of the plasma in the Solar System. As a result, new knowledge can be obtained on the propagation of disturbances from the atmosphere into the ionosphere, including the propagation of wave disturbances, the nature of which has not been studied to date.

\section{ACKNOWLEDGMENTS}

The work was performed within the framework of a state task and was partially supported by the Program of the Russian Academy of Sciences No. 28.

\section{REFERENCES}

1. Armand NA, Gulyaev YuV, Gavrik AL, Efimov AI, Matyugov SS, Pavel'ev AG, Savich NA, Samoznaev LN, Smirnov VM, Yakovlev OI. Results of solar wind and planetary ionosphere research using radiophysical methods. Physics-Uspekhi, 2010, 53 (5):517-523.

2. Fjeldbo G, Kliore AJ, Eshleman VR. The Neutral Atmosphere. The Astronomical Journal, 1971, 76 (2):123-140. 
3. Fjeldbo G, Seidel B, Sweetnam D, Hovard T. The Mariner 10 Radio Occultation Measurement of the Ionosphere of Venus. Journal of the Atmospheric Sciences, 1975, 32 (2):1232-1236.

4. Savich ON., Gavrik AL., Samoznaev LN. Analysis of diffusion processes in the daytime Venus ionosphere according to radio emission data of Venus-9,10 satellites. Space research, 1983, 21 (5):737-745.

5. Brace LH, Kliore AJ. The structure of the Venus ionosphere. Space Science Reviens, 1991, 55:81-163.

6. Gavrik AL, Gavrik YuA, Kopnina TF, Samoznaev LN. Variations of the amplitudes and frequencies of coherent radio signals when radiating Venus's daytime ionosphere. Radioengineering and electronics, 2010, 55(3):277-284.

7. Jenkins JM, Steffes PG, Hinson DP et al. Magellan spacecraft: 2. Results from the October 1991 experiments. Icarus, 1994,110:79-94.

8. Gérard JC, Bougher SW, López-Valverde MA, Pätzold M, Drossart P, Piccioni G. Aeronomy of the Venus Upper Atmosphere. Space Sci. Rev., 2017, 212 (3-4):1617-1683.

9. Imamura T, Ando H, Tellmann S, Pätzold M, Häusler B, Yamazaki A, Sato TM, Noguchi K, Futaana Y, Oschlisniok J, Limaye S, Choudhary RK, Murata Y, Takeuchi H, Hirose C, Ichikawa T, Toda T, Tomiki A, Abe T, Yamamoto Z, Noda $H$, Iwata $T$, Murakami S, Satoh T, Fukuhara T, Ogohara K, Sugiyama K, Kashimura H, Ohtsuki S, Takagi S, Yamamoto Y, Hirata N, Hashimoto GL, Yamada M, Suzuki M, Ishii N, Hayashiyama T, Lee YJ, Nakamura M. Venus orbiter mission Akatsuki. Earth, Planets and Space, 2017, 69 (1):137.

10. Glaze LS, Wilson CF, Zasova LV, Nakamura M, Limaye S. Future of Venus Research and Exploration. Space Sci Rev., 2018, 214:89.

11. Gavrik AL, Gavrik YuA, Kopnina TF, Kuleshov EA. Oscillations near the lower boundary of the Venus ionosphere using radio occultation data from Venus-15,-16 satellites. Radioengineering and electronics, 2013, 58 (10):1013-1024.

12. Gavrik AL, Samoznaev LN. Analysis of the errors of the results of the radio sounding of the daytime Venus ionosphere due to its nonsphericity. Space research, 1985, 23 (1):148-15.

13. Withers P. Prediction of atmospheric properties measured by radio occultation experiments. Advances in Space Research, 2010. 46:58-73.

14. Gavrik AL, Bondarenko MI, Smyslov AA, Kopnina TF. Method of diagnostics of layered structures in the atmosphere and ionosphere according to radio sounding data. Journal of Radioelectronics [online journal. 2017, 11:7; http://jre.cplire.ru/jre/nov17/9/text. pdf.

15. Yakovlev OI. The propagation of radio waves in space. Moscow, Nauka Publ., 1985, 214 p. 